\title{
Numerical simulation of a tuneable reversible flow design for practical ACET devices
}

\author{
Thomas Lijnse ${ }^{4}\left(\mathbb{D} \cdot\right.$ Stirling Cenaiko ${ }^{2,3} \cdot$ Colin Dalton ${ }^{1,4}(\mathbb{D}$
}

Received: 2 December 2019 / Accepted: 22 January 2020 / Published online: 30 January 2020

(C) The Author(s) 2020 OPEN

\begin{abstract}
Alternating Current Electrothermal (ACET) micropumps are a well-documented flow induction and mixing method. This phenomenon has significant promise as a reliable microfluidic pumping method for high conductivity biofluids, such as cerebrospinal fluid, urine, or blood. Practical implementations so far have been limited by complex designs focused on maximized flow rates, typically in only one direction at a time. This paper describes a device geometry demonstrating, and quantifying for the first time, fully reversible flow, that is, going from $100 \%$ flow in one direction to fully symmetrical $100 \%$ flow in the opposite direction. This design incorporates multiple features targeted at practical fabrication and applications. The design enables fine-tuning of flow speeds via adjustable signal strengths in a unique manner compared to traditional ACET devices. A full numerical simulation of this device has been performed within this work. Additionally, this paper reports several methods for increasing usability of ACET devices, including proposing coatings to prevent electrolysis and increase flow rates without the risk of fluid reactions, manufacturing methods for ease of handling, and specific device parameters for implementation in microdevices. The development of an ACET device that can precisely and efficiently pump and extract fluids allows for new applications in integrated biological systems and monitoring devices.
\end{abstract}

Keywords Electrokinetics Electrothermal $\cdot$ Lab-on-a-chip $\cdot$ Microfluidics $\cdot$ Micropump $\cdot$ Microfabrication

\section{Introduction}

Miniaturization of biomedical lab-on-a-chip devices is key in transitioning to point-of-care medicine, by reducing power consumption, sample size, analysis time and device footprint. This enables precise analytics without the use of large benchtop systems. The AC Electrothermal (ACET) technique has shown high effectiveness in the transport and mixing of conductive biofluids, required for miniaturized lab-on-a-chip systems $[3,19,20,25]$. Through the production of vortices in the fluid, ACET is also capable of simultaneously mixing and transporting fluids $[3,19]$. ACET is the only electrokinetic based technique capable of interacting with high conductivity fluids $(>1 \mathrm{~S} / \mathrm{m})$ at relatively low voltages $\left(<10 \mathrm{~V}_{\mathrm{RMS}}\right)$ [28], making it a practical biofluid pumping technique when compared to other electrokinetic techniques, that typically require tens to hundreds of volts $[12,14,18]$.

ACET devices have seen applications in microfluidics focusing on the mixing of solutions for cell aggregation, cell sorting, and pharmaceutical testing $[3,9,11,13]$, with further applications in miniaturized droplet mixing, bioassays, open channel microfluidics, and contact line dynamics [5-9, 21, 22]. Unfortunately, however, these applications typically rely on external monitoring systems, negating the usefulness of the miniaturization. To increase versatility,

Thomas Lijnse, thomas.lijnse@ucalgary.ca | ${ }^{1}$ Electrical and Computer Engineering Department, University of Calgary, Calgary, Canada. ${ }^{2}$ Chemical and Petroleum Engineering Department, University of Calgary, Calgary, Canada. ${ }^{3}$ Centre for Bioengineering Research and Education, University of Calgary, Calgary, Canada. ${ }^{4}$ Biomedical Engineering Graduate Program, University of Calgary, Calgary, Canada. 
ACET pumps should have a fine degree of control over both flow and directionality. Flow reversal has been documented in relatively high conductivity solutions $(0.02 \mathrm{~S} / \mathrm{m})[10]$ exclusively through the use of a $1 \mathrm{kHz}$ signal at $5.3 \mathrm{~V}$, which may reorient the flow through the AC electroosmotic (ACEO) effect. While an interesting phenomenon, this flow reversal is highly specific to set conditions and device geometry and does not lend itself to practical device implementation. The current work reports a geometry applicable to fully controllable bi-directional, practical ACET micropump fabrication and characterizes the flow reversibility outcomes. While Vafai et al. propose a similar design, and state reversible flow direction was possible, it is reported without any quantification. In this work characterization of the direction switching and the use of differential voltages on pairs of control electrodes is unique, particularly when considering most reversible phenomenon have been investigated in proximity to single control electrodes [10, 26].

\subsection{Theory}

ACET Theory is well documented $[1,2]$ and is summarized as follows. ACET flow is derived from the energy balance equation in which an electric field applied across a conductive fluid induces joule heating. We see the equation for the temperature field in the solution through Eq. 1 [4].

$k \nabla^{2} T+\sigma E^{2}=0$

where $k$ is the thermal conductivity of the solution, $\sigma$ is the electrical conductivity, $E$ represents the electric field, $\sigma E^{2}$ is the Joule heating term, and $\nabla T$ is the thermal gradient. Additionally, the electric field in a non-changing dielectric can be modelled using Eq. 2 .

$\nabla^{2} \phi=0, E=-\nabla \phi$

where $\phi$ represents field potential. By combining Eqs. 1 and 2 into the Navier-Stokes equation, assuming a laminar incompressible fluid flow and negligible effects due to gravity or buoyancy, we have the resultant Eq. 3 detailing the effects of AC electrothermal force on flow $[1,4]$.

$-\nabla p+\eta \nabla^{2} u+F_{e}=0$

where $p$ represents the pressure vector field, $\eta$ represents dynamic viscosity, $u$ represents the fluid velocity field and $F_{e}$ represents electrothermal force, which is given in time averaged form below [20]. It is important to note that $\eta$ will vary with temperature fluctuations.

$F_{E}=\frac{1}{2} \frac{\epsilon(\alpha-\beta)}{1+(\omega \tau)^{2}}(\nabla T \cdot E) E-\frac{1}{4} \epsilon \alpha|E|^{2} \nabla T$
The equation for electrothermal force assumes the change with respect to temperature for electric permittivity, given by $\alpha$, and conductivity, given by $\beta$, are $-0.4 \%$ $\mathrm{K}^{-1}$ and $2 \% \mathrm{~K}^{-1}$ respectively [20].

\section{Materials and methods}

The simulations presented here are built on experimentally validated simulation work and modifications to previously published electrode designs $[17,19,23,24]$. The simulations are completed in a standard Multiphysics simulation software (COMSOL). Potassium Chloride $(\mathrm{KCl})$ is used as the working fluid-with an electrical conductivity equal to $0.224 \mathrm{~S} / \mathrm{m}$ and a temperature dependent dynamic viscosity [17]. $\mathrm{KCl}$ is chosen as an appropriate simulation material due to its conductivity of a similar magnitude to that of biofluids, and its prevalence in prior reported work for ease of comparison [11]. As the ACET force proportionally increases with fluid conductivity, higher conductivity biofluids $(>1 \mathrm{~S} / \mathrm{m})$ will experience higher flow rates $[4,20]$. Figure $1 \mathrm{a}-\mathrm{c}$ shows the boundary conditions and new electrode geometry, featuring two thin electrodes adjacent to either side of a wide electrode on both the top and bottom of a microfluidic channel. These boundary conditions, described in the caption of Fig. 1 and Table 1, were chosen to simulate realistic conditions in which an ACET electrode micropump will operate, including normal ambient temperatures and no slip conditions at the walls. A zero pressure point is chosen at the fluid inlet, as it is required for accurate Multiphysics simulations, and these ACET systems will not be subject to external forces [29]. Figure 2 shows a top down view of ACET electrodes fabricated on a silicon substrate for reference. These three electrodes can be treated as one entity and repeated as functional units. Multiple substrates and substrate thicknesses are analyzed for thermal properties and overall effect on fluid flow, keeping in mind a focus on practical microfabrication and implementation. Specific property values used in the simulations are shown in detail in Table 1. Electrode spacing parameters were selected, and varied where appropriate, from previous work regarding channel electrode geometry optimization [17].

A 2D model was used to study the bidirectional flow rather than a 3D model, as it has been shown to have a negligible difference on results when compared to experimental results $[16,17]$. An automatic triagonal mesh size "extra fine" was applied in COMSOL to the entire model, as the "extremely fine" mesh size yielded a difference in flow of only $0.158 \%$ from the "extra fine" mesh. The "extra fine" mesh produced more than 16,000 elements in each simulation. 


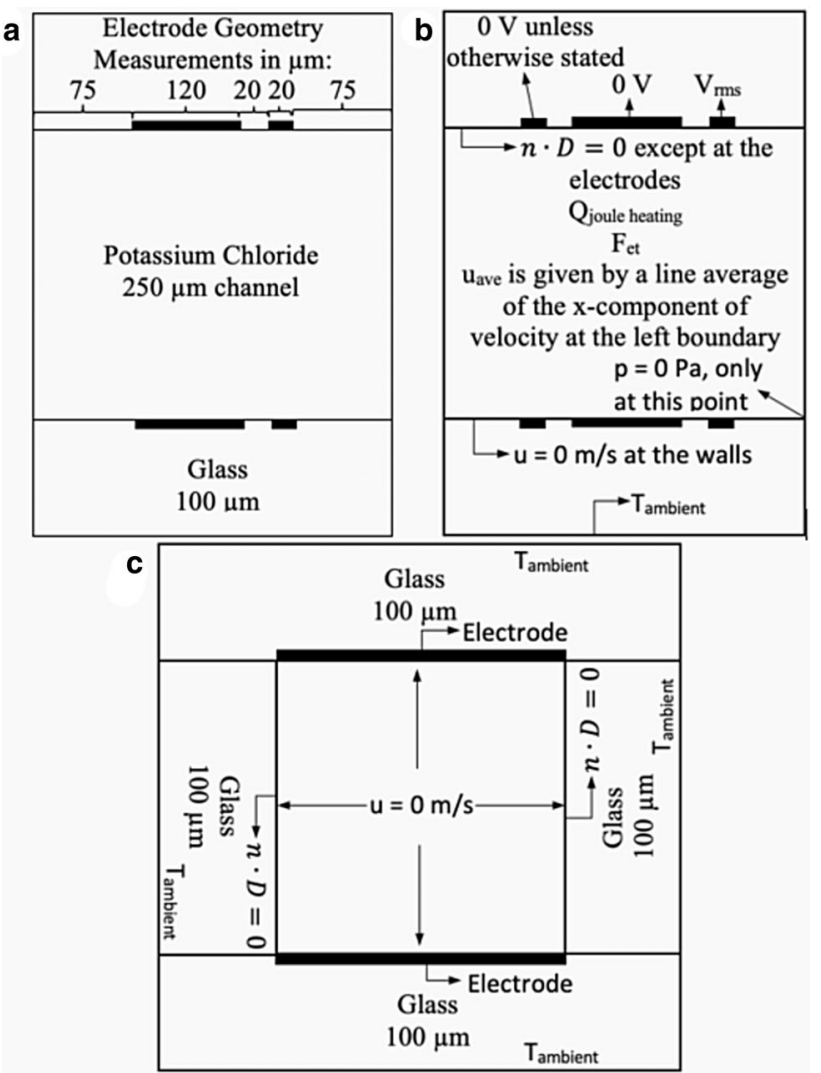

Fig. 1 The boundary conditions and parameters applied to the electrostatics, heat transfer, and laminar flow modules in a 2D COMSOL (v5.4) model. The model is completely symmetrical about the $x-z$ plane, excluding the pressure point constraint applied to the bottom right corner of the channel as shown in Fig. 3b. a The physical geometry of the model in the $x-y$ plane. The electrodes are shown as solid black rectangles, but are assumed to be completely planar in the COMSOL model for the purposes of this paper. $\mathbf{b}$ The boundary conditions applied to the model in the $x-y$ plane. Periodic conditions are applied to the left and right boundaries of the substrate (only applicable for heat transfer) and the channel. $\mathbf{c}$ Is a cross-sectional view of the geometry in the $y-z$ plane

\subsection{Practical fabrication}

The simulations performed in this work use an aligned ACET electrode array on both the top and bottom of the channel. This has been previously shown to increase channel flow rates by $107 \%$ [17] when compared to traditional ACET geometry devices. While alignment of both top and bottom channels poses a significant challenge, misalignments can be utilized to provide significant fluid mixing without sacrificing flow rates, and has been reported in prior work [19]. Simultaneous mixing is a desirable outcome in microfluidic devices $[3,12,27]$.

As will be discussed later in the work, the electrodes can be fabricated using standard photolithography and etching techniques, with the potential application of a dielectric coating performed using a basic shadowmask
Table 1 Boundary conditions used in COMSOL simulations

\begin{tabular}{ll}
\hline Property & Simulation value \\
\hline Input signal & \\
Frequency & $100 \mathrm{kHz}$ \\
$\mathrm{V}_{\text {rms }}$ & $7 \mathrm{~V}$ \\
Ambient temperature & $293.15 \mathrm{~K}$ \\
Fluid: $\mathrm{KCl}$ & \\
Viscosity at $293.15 \mathrm{~K}$ & $1.05 \mathrm{e}-3 \mathrm{Ns} / \mathrm{m}^{2}$ \\
Heat capacity at constant pressure & $4181 \mathrm{~J} / \mathrm{kgK}$ \\
Density & $999 \mathrm{~kg} / \mathrm{m}^{3}$ \\
Thermal conductivity & $0.598 \mathrm{~W} / \mathrm{mK}$ \\
Ratio of specific heats & 1 \\
Electrical conductivity & $0.224 \mathrm{~S} / \mathrm{m}$ \\
Relative permittivity & 80.2 \\
Substrate: glass & \\
Heat capacity at constant pressure & $480 \mathrm{~J} / \mathrm{kgK}$ \\
Density & $2200 \mathrm{~kg} / \mathrm{m}^{3}$ \\
\hline
\end{tabular}

Full settings and boundary conditions used for the COMSOL simulations of electrodes, materials, and microchannel

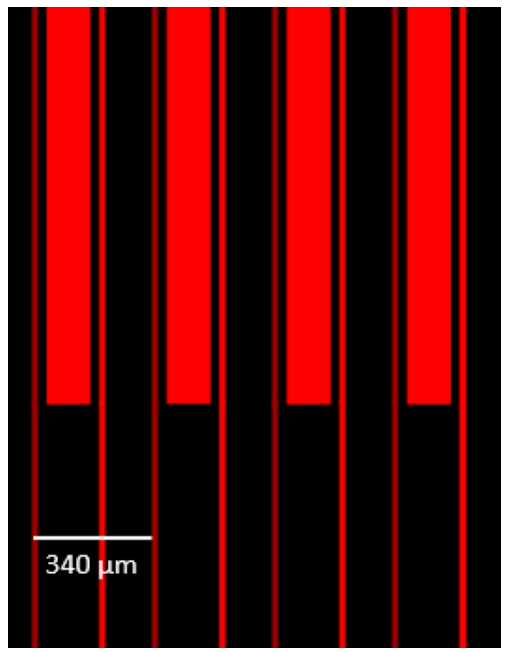

Fig. 2 A planar, top down, view of the ACET electrode design, dimensions. In channel, fluid will flow from left to right, or from right to left

over the electrode area of interest. Ultimately the substrates selected for simulation were chosen based on ease of manufacturability, and practical handling considerations for end-stage fabricated devices.

The external electronics to an ACET device, while currently quite large for use in lab settings, can be miniaturized through the use of a microcontroller with high quality DACs, charge pump converters, and filtering electronics. While the design of such a system is feasible and applicable to practical fabrication, it would require 
extensive experimental testing and falls outside the scope of this work.

\section{Results and discussion}

The electrode geometry presented here resulted in a maximum flow rate in either direction as selected in the microfluidic channel. In addition, the flow rate direction can be reversed quickly by alternating the location of the input signal with respect to the major ground plane electrode. This is not possible with traditional ACET electrodes, where the flow direction is in one direction only and set at the start of experiments by the electrode physical geometry. Being able to switch between flow directions, and have maximum flow rate in either direction, has not been previously shown. Traditional ACET devices have flows in the range of $60-100 \mu \mathrm{m} / \mathrm{s}[22,24,27]$, however these flows are typically exaggerated through thermal biasing, which is damaging to biofluids, or for high voltage applications $\left(>10 V_{\text {RMS }}\right.$ ). We report flow rates, calculated using the methods described in Fig. 1b, of up to $107.51 \mu \mathrm{m} / \mathrm{s}$ with the temperature remaining within a range acceptable for biofluid processing without damage-299.27 K at max velocity - and at low voltages $\left(<10 \mathrm{~V}_{\mathrm{RMS}}\right)$ which will not negatively interact with biofluids or cause degradation of electrodes [17]. The modifications made to the electrode geometry, substrate material and thickness, and electrodes on both the top and bottom of the channel increases flow rates, and thus practicality of the micropump. These flow rates can be dramatically increased by increasing channel height [17], but for the sake of consistency in comparison of flow rates between journals, standard channel dimensions have been used, namely a 250 micron channel height.

As expected, and seen in Fig. 3a, b the flow profiles are symmetrical in either direction. Full flow reversal is obtained in $1.31 \mathrm{~s}$, and temperature equilibrium in $0.66 \mathrm{~s}$, when assuming $1 \mathrm{~ms}$ to shift location of the applied voltage between the electrodes. In addition to the implementation of fully controllable, bidirectional flow, an efficiency increase of $\sim 1 \%$ from dual-sided ACET electrode geometries $[17,19]$ is observed when the second small electrode is held at $0 \mathrm{~V}$. This may be due to the large-small ground

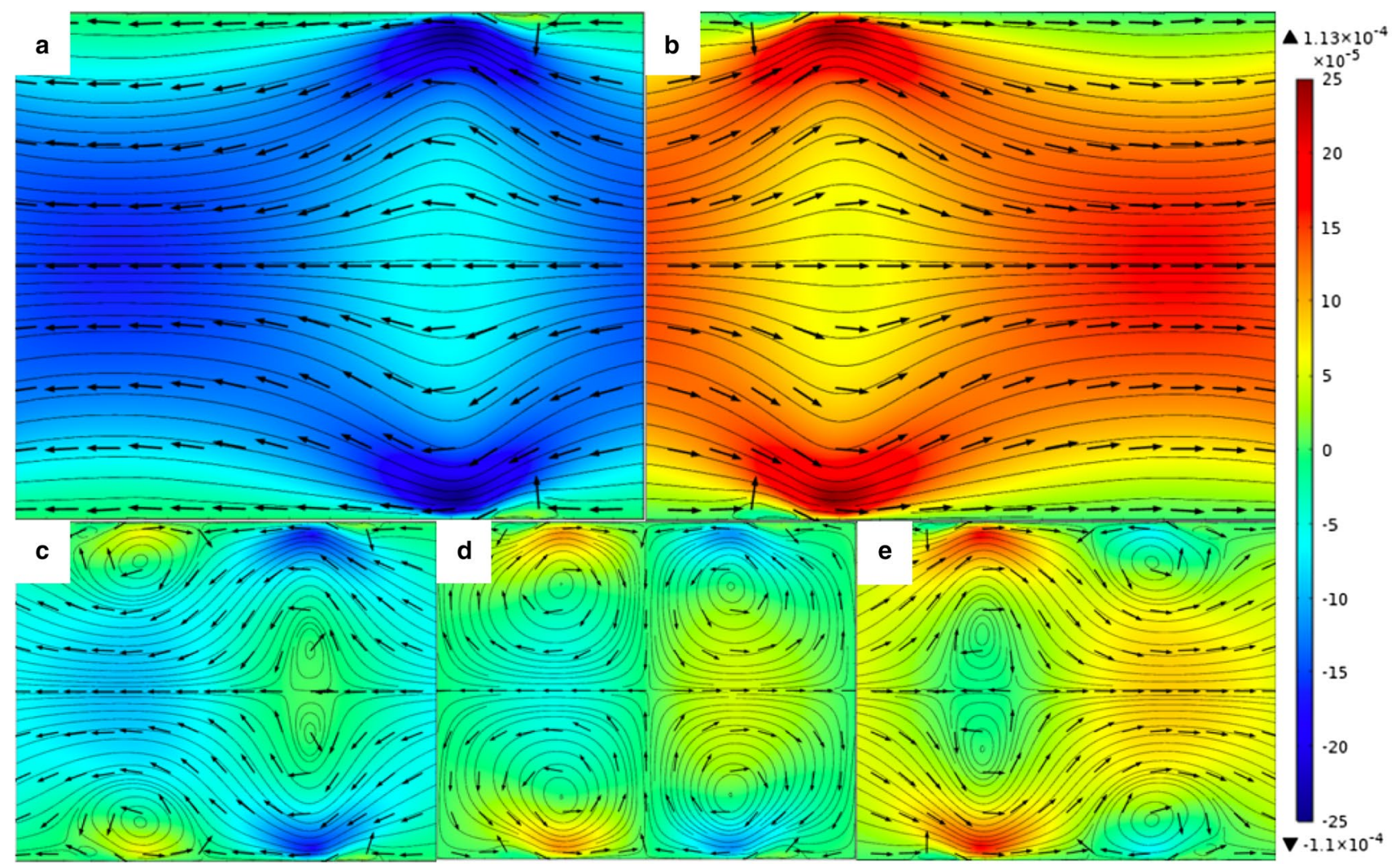

Fig. 3 The velocity profile in the $x$-direction and flow streamlines resulting from the $2 \mathrm{D}$ simulation. a Results in a net flow to the left (in the negative direction by convention), while $\mathbf{b}$ results in a net flow to the right (in the positive direction). In a the rightmost small electrode is set to $V_{r m s}$ and in $\mathbf{b}$ the leftmost small electrode is set to $V_{r m s}$. These figures show that the flow is $100 \%$ reversible. With the change in voltage happening between 2 and $2.001 \mathrm{~s}, \mathbf{c}-\mathbf{e}$ show the process of the flow being reversed at $2.10 \mathrm{~s}, 2.18 \mathrm{~s}$, and $2.26 \mathrm{~s}$ respectively. f Represents the colour scale used in $\mathbf{a}-\mathbf{e}$, where the speeds are shown in $\mathrm{m} / \mathrm{s}$ 
electrode pair acting as one large "virtual ground" electrode, leading to a more condensed electric field, with less variance at further distances from the electrode. This effect may cause slightly higher flows as a more uniform electric field at further distances from the source electrode will induce less turbulence. Experimental testing to confirm this is required. And will be the subject of future work.

ACET electrodes in experimental models do experience electrolytic degradation [18, 24, 25], and as such, the coating of electrodes in a protective or sacrificial material becomes necessary at higher voltages. A commonly used electrode insulation material is Silicon Dioxide $\left(\mathrm{SiO}_{2}\right)$ or similar silicon base materials due to their insulating properties $[15,24]$. We investigated electrode insulation parameters and the corresponding effect on channel flow, which revealed a relationship primarily based on the thermal conductivity and electric permittivity of the coating materials. As seen in Fig. 4 a non-conductive material with low thermal conductivity and high electric permittivity is ideal. In Table 2 a brief list of some materials capable of being used in microfabrication are compared, focusing on metal oxides, which have typically low thermal conductivities, high relative permittivities and are non-conductive. Figure 5 shows the channel flow values observed through a number of highly relevant coating materials compared to flow in a channel without a coating. Importantly, some of the simulated materials are used to illustrate the concept, such as Lead Oxide, which is actually an infeasible

Coating Thermal Conductivity and Relative Permittivity Effect on Flow Rate

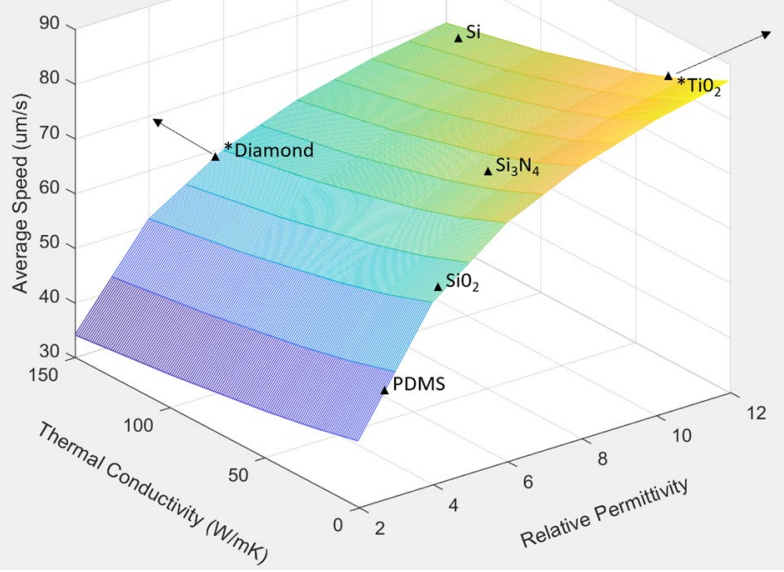

Fig. 4 Results from a 2D simulation of a hypothetical, $100 \mathrm{~nm}$ thick insulating material at different values of $k_{\text {iso }}$ and $\varepsilon$. Asterisk indicates the material properties extend beyond the boundary of the axis. Flow measurements are built on assumed planar topology of electrodes as previously shown in Fig. 1 . This work is indicative of the potential of a dielectric coating to maintain approximate channel flow rates without risk of damage to the driving electrodes
Table 2 Material properties of insulative coatings

\begin{tabular}{lll}
\hline Material & $\begin{array}{l}\text { Thermal conductivity }(\mathrm{W} / \\
\mathrm{mK})\end{array}$ & $\begin{array}{l}\text { Relative } \\
\text { permittivity } \\
\left(\epsilon_{0}\right)\end{array}$ \\
\hline $\mathrm{SiO}_{2}$ & 1.4 & 4.2 \\
$\mathrm{Si}_{3} \mathrm{~N}_{4}$ & 30 & $7-8$ \\
Diamond & 2500 & 5.7 \\
$\mathrm{TiO}_{2}$ & $4.8-11.8$ & 85 \\
$\mathrm{Si}$ & 130 & 11.7 \\
PDMS & 0.16 & 2.75 \\
\hline
\end{tabular}

Material properties of various proposed simulation materials used in COMSOL simulations. Values are sourced from internal COMSOL material properties database

material for use with biological systems, as it is not biocompatible. Of note, is that the materials which provide the highest flow rates are titanium oxide compounds. As ACET devices are typically low voltage $\left(<10 \mathrm{~V}_{\mathrm{RMS}}\right)$ we do not predict issues regarding dielectric breakdown in high field strengths. Conversely, the alternating signal effects on a capacitive dielectric may have profound impacts on channel flow rates that are not addressed by the COMSOL model, and as such, further simulation and experimental work must be performed. Ultimately, the use of a dielectric coating will allow for increased channel voltages, leading to higher flow rates with significantly less potential for electrochemical interference between channel fluid and electrodes, allowing for more relevant and more feasible ACET pumps to be created.

Multiple sets of electrode geometry paired with different substrate parameters were then analyzed with respect to effect on temperature and flow fluctuations, as shown in Fig. 6 and Table 3. It was found that silicon substrates yielded a decrease in flow to $43.94 \mu \mathrm{m} / \mathrm{s}$ (59.1\% decrease)

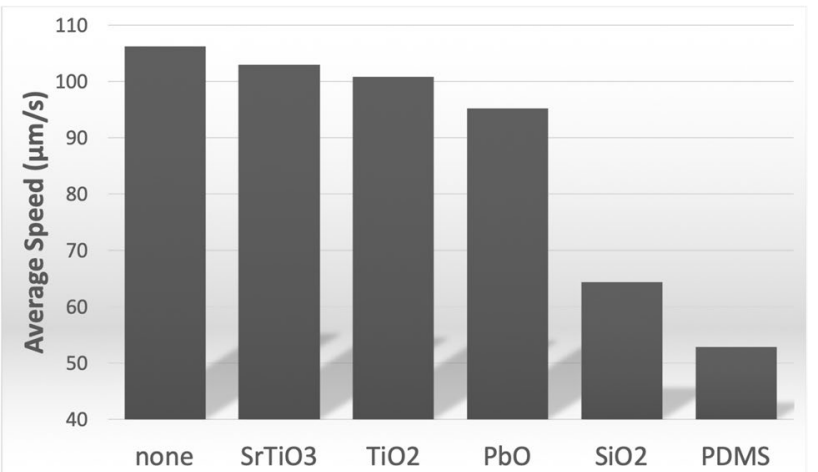

Fig. 5 Results from a 2D COMSOL simulation of $100 \mathrm{~nm}$ coatings of various metal oxides and a commonly used microchannel polymer, PDMS, against the baseline flow rate of no coating. The initial results show higher flow rates associated with metal oxides, as opposed to polymers or non-metal oxides 


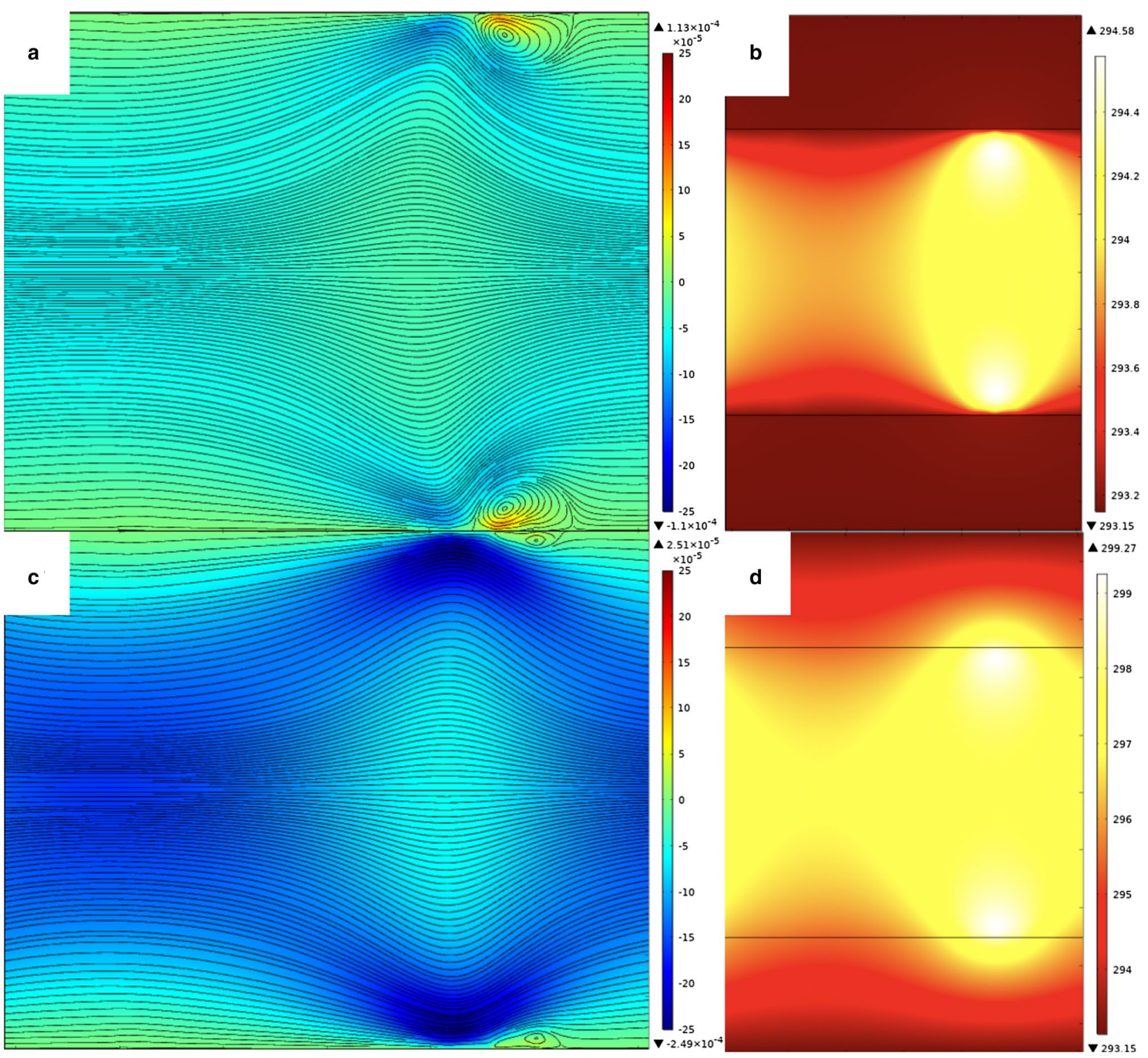

Fig. 6 These images illustrate the effect that the substrate material can have on velocity, using a silicon substrate. a The $x$-component of velocity and streamlines obtained, using the same colour scale depicted in Fig. 2f, while $\mathbf{b}$ is the temperature field of the silicon model with a maximum temperature of 294.58 K. c, d The velocity profile and the temperature field of the model with the glass substrate, for comparison. Simulations of the glass substrate give a maximum temperature of $299.27 \mathrm{~K}$ and in temperature to $294.58 \mathrm{~K}$ (1.6\% decrease), which is consistent with previous published ACET work [18]. The $100 \mu \mathrm{m}$ thick glass used as a base substrate provides promising results for flow and temperature, but is inherently delicate and difficult to work with in practical devices. A more practical $500 \mu \mathrm{m}$ glass substrate increases flow by $31.3-141.11 \mu \mathrm{m} / \mathrm{s}$, but raises the temperature to $312.45 \mathrm{~K}$, which is near the threshold of damaging biofluids. Coating a $500 \mu \mathrm{m}$ copper layer to the bottom of the $100 \mu \mathrm{m}$ glass layer marginally increases the flow rate $(<0.1 \%)$ and temperature $(<0.1 \%)$ while making a practical, fabricated device easier to handle, removing the need for a thin, brittle, glass substrate, while maintaining safe operating conditions for biofluid applications.

The geometry of two small voltage-adjustable electrodes opens the possibility of changing one electrode to be $V_{r m s}$ and the opposing electrode to be $n V_{r m s}$ where $n$ is some fractional value between 0 and 1 , enabling precise tuning of flow speeds. Setting $n=0.5$ results in a flow of $99.3 \mu \mathrm{m} / \mathrm{s}$, corresponding to an $8 \%$ reduction in flow. Setting $n=1$ will reduce the speed to near zero, as the system becomes a symmetrical heater. We report the relationship

\section{SN Applied Sciences}


Table 3 Geometries and parameters simulated

\begin{tabular}{lllllll}
\hline Configuration $(\mu \mathrm{m})$ & $\begin{array}{l}\text { Configuration } \\
(\mu \mathrm{m})\end{array}$ & Substrate material & $\begin{array}{l}\text { Substrate thick- } \\
\text { ness }(\mu \mathrm{m})\end{array}$ & $\mathrm{u}_{\mathrm{ave}}(\mu \mathrm{m} / \mathrm{s})$ & Flow rate $\left(\mu \mathrm{m}^{2} / \mathrm{s}\right)$ & $\mathrm{T}_{\text {max }}(\mathrm{K})$ \\
\hline $20 / 120$ & 250 & Glass & 100 & 106.34 & 26586 & 299.05 \\
$20 / 120 / 20$ & 250 & Glass & 100 & 107.51 & 26878 & 299.27 \\
$20 / 120 / 20$ & 250 & Glass & 500 & 141.11 & 35278 & 20642 \\
$20 / 120 / 20$ & 200 & Glass & 100 & 103.21 & 10985 & 29.45 \\
$20 / 120 / 20$ & 250 & Silicon & 100 & 104.76 & 26190 & 294.58 \\
$20 / 80 / 20$ & 250 & Glass & 100 & & 299.03 \\
\hline
\end{tabular}

The configuration column shows the widths of the electrodes in a repeating unit, where there is a $20 \mu \mathrm{m}$ gap between electrodes and the total repeating unit is $310 \mu \mathrm{m}$ in length (see Fig. 1 for more information). Flow rates are obtained by multiplying the average speed by the channel depth, and are useful for comparing the volumetric flow rates that would be obtained in 3D

between $n$ and fluid velocity, seen in Fig. 7. By adjusting $n$, the micropump flow rate becomes adjustable to a high degree of accuracy. Commercially available microfluidic pumping systems typically have both coarse and fine flow adjustment capabilities. With the device reported here, voltage adjustments to the primary working electrode provide coarse fluid control, and voltage adjustments to the secondary small electrode provide fine fluid control. Ultimately these parameters result in an ACET device that can be considered finely tuneable and highly controllable, which separates this design from standard ACET configurations, which cannot precisely control flow rates based off input voltage to the traditional two electrodes.

\section{Conclusion}

This work reports an ACET electrode configuration that enables full control over bidirectional flow in a microchannel and, primarily, the details of operation surrounding the device that enable implementation in future practical systems. The paired symmetrical electrode design additionally allows for fine-tuning of flow rates for further applications in precision devices, which has not been shown to be feasible with high accuracy using traditional ACET designs. The extent to which flow is reversible has been characterized, indicating applications in future microfluidic pumping/extracting systems. Simulations of electrode coatings show a practical method to deal with existing issues surrounding electrolysis of electrodes, leading to increased long-term usability, and safer operation of highspeed channel flows without risk of fluid-electrode interaction. The ability for ACET to be used for both pumping and extracting purposes without the need for physical changes in electrode design or separate devices will significantly increase the usefulness and applicability of ACET in future biomicrofluidic applications.
Fig. 7 A graph showing how the velocity varies with a voltage applied to the second small electrode, while keeping the first small electrode at $7 \mathrm{~V}$. This differential in voltages from the central ground electrode will cause the field over the electrode to become slightly more symmetrical, both reducing speed and improving mixing. As this relationship follows the curve shown above, this can be used to fine tune the speed of the fluid in the channel with a higher degree of accuracy than simply by changing the input electrode voltage, as has been described in other works [20]
Applying a Voltage to the Second Small Electrode to Control the Velocity

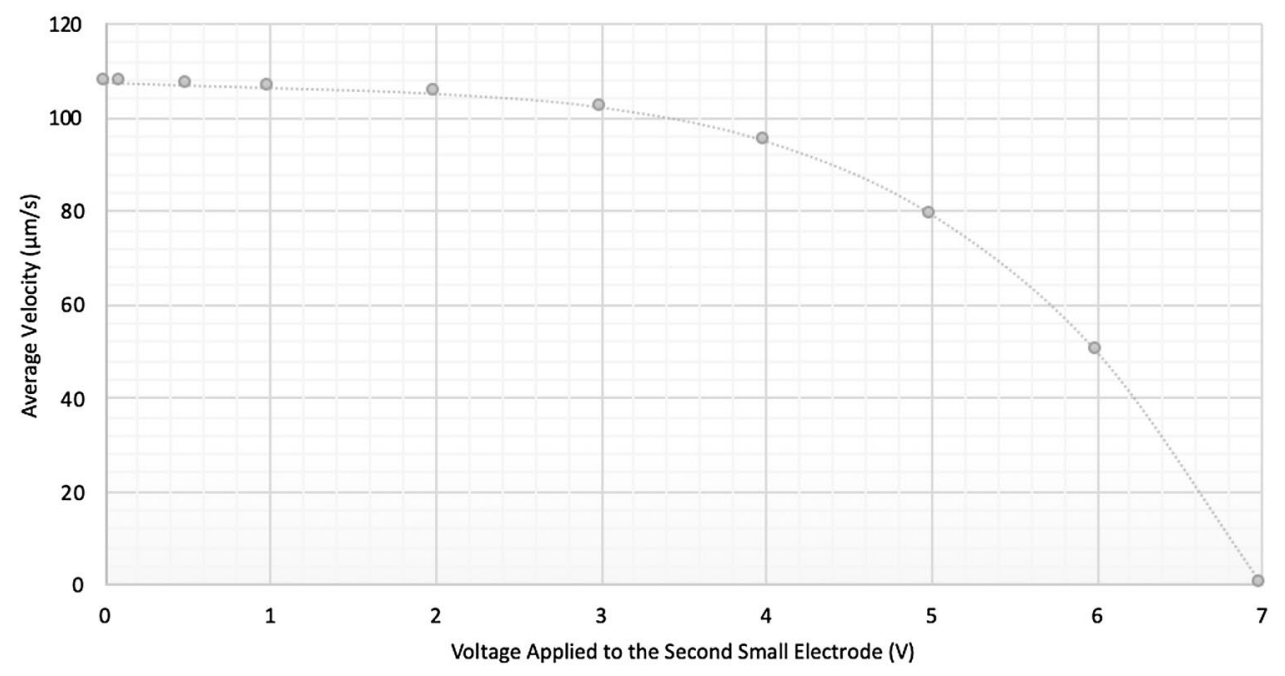


Acknowledgements The authors would like to thank CMC microsystems and COMSOL Inc. for providing the software used in these simulations, as well as Alberta Innovates and the National Science and Engineering Research Council of Canada (NSERC) for financial support.

\section{Compliance with ethical standards}

Conflict of interest The authors have no conflict of interest to declare.

Open Access This article is licensed under a Creative Commons Attribution 4.0 International License, which permits use, sharing, adaptation, distribution and reproduction in any medium or format, as long as you give appropriate credit to the original author(s) and the source, provide a link to the Creative Commons licence, and indicate if changes were made. The images or other third party material in this article are included in the article's Creative Commons licence, unless indicated otherwise in a credit line to the material. If material is not included in the article's Creative Commons licence and your intended use is not permitted by statutory regulation or exceeds the permitted use, you will need to obtain permission directly from the copyright holder. To view a copy of this licence, visit http://creativecommons .org/licenses/by/4.0/.

\section{References}

1. Ajdari A (2000) Pumping liquids using asymmetric electrode arrays. Phys Rev E 61(1):R45-R48. https://doi.org/10.1103/PhysR evE.61.R45

2. Feldman HC, Sigurdson M, Meinhart CD (2007) AC electrothermal enhancement of heterogeneous assays in microfluidics. Lab Chip 7(11):1553-1559. https://doi.org/10.1039/b706745c

3. Gao X, Li Y (2018) Biofluid pumping and mixing by an AC electrothermal micropump embedded with a spiral microelectrode pair in a cylindrical microchannel. Electrophoresis 39(24):31563170. https://doi.org/10.1002/elps.201800162

4. Green NG, Ramos A, González A, Castellanos A, Morgan H (2001) Electrothermally induced fluid flow on microelectrodes. J Electrost Sel Pap Int Workshop Electr Conduct Convect Breakdown Fluids 53(2):71-87. https://doi.org/10.1016/S0304 -3886(01)00132-2

5. Kunti G, Bhattacharya A, Chakraborty S (2019) Strong rotating flow in stationary droplets in low power budget using wire electrode configuration. Electrophoresis 40(22):2971-2978. https:// doi.org/10.1002/elps.201900272

6. Kunti G, Bhattacharya A, Chakraborty S (2019) Interfacial dynamics of immiscible binary fluids through ordered porous media: the interplay of thermal and electric fields. Phys Fluids 31(3):032002. https://doi.org/10.1063/1.5080301

7. Kunti G, Dhar J, Bhattacharya A, Chakraborty S (2019) Directionally controlled open channel microfluidics. Phys Fluids 31(9):092003. https://doi.org/10.1063/1.5118728

8. Kunti G, Mondal PK, Bhattacharya A, Chakraborty S (2018) Electrothermally modulated contact line dynamics of a binary fluid in a patterned fluidic environment. Phys Fluids 30(9):092005. https://doi.org/10.1063/1.5044268

9. Lang Q, Ren Y, Hobson D, Tao Y, Hou L, Jia Y, Hu Q, Liu J, Zhao $X$, Jiang $H$ (2016) In-plane microvortices micromixer-based AC electrothermal for testing drug induced death of tumor cells. Biomicrofluidics. https://doi.org/10.1063/1.4967455
10. Lian M, Wu J (2009) Microfluidic flow reversal at low frequency by AC electrothermal effect. Microfluid Nanofluid 7(6):757. https ://doi.org/10.1007/s10404-009-0433-6

11. Lian MI, Islam N, Wu J (2007) AC electrothermal manipulation of conductive fluids and particles for lab-chip applications. IET Nanobiotechnol 1(3):36-43. https://doi.org/10.1049/ietnbt:20060022

12. Meng J, Li S, Li J, Yu C, Wei C, Dai S (2018) AC electrothermal mixing for high conductive biofluids by arc-electrodes. J Micromech Microeng 28(6):065004. https://doi.org/10.1088/1361-6439/ aab39b

13. Mi S, Li B, Yi X, Xu Y, Du Z, Yang S, Li W, Sun W (2018) An AC electrothermal self-circulating system with a minimalist process to construct a biomimetic liver lobule model for drug testing. RSC Adv 8(65):36987-36998. https://doi.org/10.1039/C8RA03724H

14. Oh J, Hart R, Capurro J, Noh HM (2009) Comprehensive analysis of particle motion under non-uniform AC electric fields in a microchannel. Lab Chip 9(1):62-78. https://doi.org/10.1039/ B801594E

15. Park S, Yossifon G (2018) Active control of ion transport within a nanofluidic system. Available: arXiv:1803.03329

16. Salari A, Navi M, Dalton C (2014) AC electrothermal micropump for biofluidic applications using numerous microelectrode pairs. In: 2014 IEEE conference on electrical insulation and dielectric phenomena (CEIDP), pp 1-4. https://doi.org/10.1109/CEIDP .2014.6995870

17. Salari A, Navi M, Dalton C (2015) A novel alternating current multiple array electrothermal micropump for lab-on-a-chip applications. Biomicrofluidics. https://doi.org/10.1063/1.4907673

18. Salari A, Dalton C (2014) Fast biofluid transport of high conductive liquids using $\mathrm{AC}$ electrothermal phenomenon, a study on substrate characteristics. In: 2014 COMSOL conference. https:// doi.org/10.13140/2.1.2183.0085

19. Salari A, Dalton C (2019) Simultaneous pumping and mixing of biological fluids in a double-array electrothermal microfluidic device. Micromachines. https://doi.org/10.3390/mi10020092

20. Salari A, Navi M, Lijnse T, Dalton C (2019) AC electrothermal effect in microfluidics: a review. Micromachines 10(11):762. https://doi.org/10.3390/mi10110762

21. Selmi M, Khemiri R, Echouchene F, Belmabrouk H (2016) Electrothermal effect on the immunoassay in a microchannel of a biosensor with asymmetrical interdigitated electrodes. Appl Therm Eng 105:77-84. https://doi.org/10.1016/j.appltherma leng.2016.05.132

22. Sigurdson M, Wang D, Meinhart CD (2005) Electrothermal stirring for heterogeneous immunoassays. Lab Chip 5(12):13661373. https://doi.org/10.1039/B508224B

23. Vafaie RH, Ghavifekr HB (2017) Configurable ACET micro-manipulator for high conductive mediums by using a novel electrode engineering. Microsyst Technol 23(5):1393-1403. https://doi. org/10.1007/s00542-015-2806-y

24. Vafaie $\mathrm{RH}$, Ghavifekr HB, Van Lintel $\mathrm{H}$, Brugger J, Renaud $\mathrm{P}$ (2016) Bi-directional ACET micropump for on-chip biological applications. Electrophoresis 37(5-6):719-726. https://doi.org/10.1002/ elps.201500404

25. Wu J, Lian M, Yang K (2007) Micropumping of biofluids by alternating current electrothermal effects. Appl Phys Lett 90(23):234103. https://doi.org/10.1063/1.2746413

26. Yang K, Wu J (2008) Investigation of microflow reversal by ac electrokinetics in orthogonal electrodes for micropump design. Biomicrofluidics 2(2):024101. https://doi.org/10.1063/1.2908026

27. Yang Ng W, Goh S, Lam YC, Yang C, Rodríguez I (2009) DC-biased $\mathrm{AC}$-electroosmotic and $\mathrm{AC}$-electrothermal flow mixing in microchannels. Lab Chip 9(6):802-809. https://doi.org/10.1039/B8136 39D 
28. Yuan Q, Wu J (2013) Thermally biased AC electrokinetic pumping effect for lab-on-a-chip based delivery of biofluids. Biomed Microdevices 15(1):125-133. https://doi.org/10.1007/s1054 4-012-9694-z

29. Zhang F, Chen H, Chen B, Wu J (2016) Alternating current electrothermal micromixer with thin film resistive heaters. Adv Mech
Eng 8(5):1687814016646264. https://doi.org/10.1177/16878 14016646264

Publisher's Note Springer Nature remains neutral with regard to jurisdictional claims in published maps and institutional affiliations. 